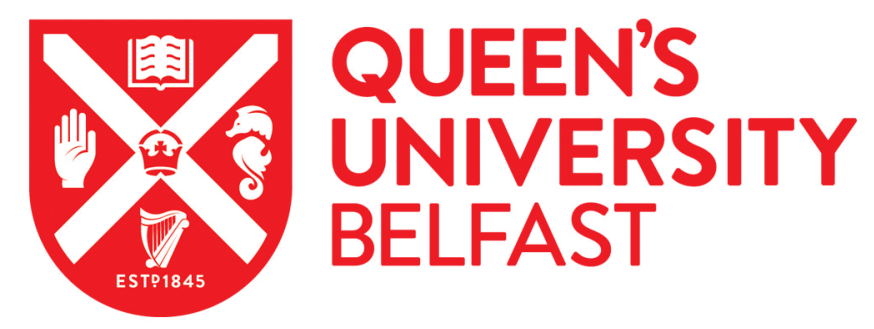

\title{
Application of modified classical numerical methods for DMPPT on Buck and Boost converters
}

Amir, A., Che, H. S., Elkhateb, A., Selvaraj, J., \& Rahim, N. A. (2018). Application of modified classical numerical methods for DMPPT on Buck and Boost converters. Solar Energy, 173, 437-448.

https://doi.org/10.1016/j.solener.2018.07.088

\section{Published in:}

Solar Energy

Document Version:

Peer reviewed version

Queen's University Belfast - Research Portal:

Link to publication record in Queen's University Belfast Research Portal

\section{Publisher rights}

Copyright 2018 Elsevier

This manuscript is distributed under a Creative Commons Attribution-NonCommercial-NoDerivs License

(https://creativecommons.org/licenses/by-nc-nd/4.0/), which permits distribution and reproduction for non-commercial purposes, provided the author and source are cited.

\section{General rights}

Copyright for the publications made accessible via the Queen's University Belfast Research Portal is retained by the author(s) and / or other copyright owners and it is a condition of accessing these publications that users recognise and abide by the legal requirements associated with these rights.

Take down policy

The Research Portal is Queen's institutional repository that provides access to Queen's research output. Every effort has been made to ensure that content in the Research Portal does not infringe any person's rights, or applicable UK laws. If you discover content in the Research Portal that you believe breaches copyright or violates any law, please contact openaccess@qub.ac.uk. 


\title{
Application of Modified Classical Numerical Methods for DMPPT on Buck and Boost Converters
}

\author{
Asim Amir a, Aamir Amir a, Che Hang Seng a, Ahmad El Khateb ${ }^{\text {b }}$, \\ Jeyraj Selvaraj ${ }^{\text {a }}$ N.A. Rahim a, c,* \\ a UM Power Energy Dedicated Advanced Centre (UMPEDAC), Level 4, Wisma R\&D, \\ University of Malaya, Jalan Pantai Baharu, 59990 Kuala Lumpur, Malaysia \\ ${ }^{b}$ School of Electronics, Electrical Engineering and Computer Science, Queen's University \\ Belfast, Belfast BT9 5AH, UK \\ ${ }^{c}$ Renewable Energy Research Group, King Abdulaziz University, Jeddah 21589, Saudi Arabia \\ *Corresponding author: nasrudin@um.edu.my
}

ARTICLE INFO

ARTICLE HISTORY:

KEYWORDS:

Digital maximum power point (DMPP) tracking (DMPPT), classical rootfinding algorithms, photovoltaic (PV), Dc-Dc converter topologies.

\begin{abstract}
Application of Classical numerical methods (CNM) for Digital maximum power point tracking (DMPPT) confronts g limited range of operation, PV array dependence and accuracy of the initial guess. In addition, the DC-DC converter cannot be treated as a black box for DMPPT, by ignoring the effects of the converter topological design and dynamics. In order to address such issues Hybrid Techniques (HT) have been presented, along with theoretical analysis to determine the optimum performance of DMPPT applications on various DC-DC converter designs. In this paper, the HT is a combination of the modified incremental conductance method (MINC) and modified CNM. An overview of MCNM, which applied to the photovoltaic (PV) application, has also been presented. The HT not only address the issues confronted by the CNM, but also remove the steady state error for the conventional MPPT technique. To measure the effectiveness of the proposed MCNM techniques, Boost, 2-Stage Switch Capacitor Based (2-SSC) Boost, and Optimum Buck Converters (OBC) have been employed. Simulation and experimental results are provided to validate the effectiveness of the proposed techniques.
\end{abstract}


Nomenclature:

\begin{tabular}{|c|c|}
\hline MPP & Maximum Power Point \\
\hline $\mathbf{P}_{\text {mpp }}$ & Power at MPP \\
\hline$\Delta \mathbf{P}$ & Change in power \\
\hline $\mathbf{V}_{\mathbf{m p p}}$ & Voltage at MPP \\
\hline$V_{\text {oc }}$ & Open circuit voltage \\
\hline$\Delta \mathbf{V}$ & Change of voltage \\
\hline$I_{\text {mpp }}$ & Current at MPP \\
\hline$\Delta I$ & Change of current \\
\hline $\mathbf{I}_{\mathbf{s h}}$ & Short circuit current \\
\hline D & Duty cycle \\
\hline$\Delta \mathbf{D}$ & Change in duty ration \\
\hline MPPT & Maximum Power Point Tracking \\
\hline e & Tolerance error \\
\hline$\Delta$ & Change \\
\hline$\Delta P_{P V} / \Delta V_{P V}$ & Change in power over change of voltage \\
\hline$\Delta \mathbf{P P V}_{\mathbf{P V}} \Delta \mathbf{I P V}$ & Change in power over change of current \\
\hline $\mathbf{D}_{\max }$ & Predefined maximum limit for the duty cycle \\
\hline$\Delta P_{P V} / \Delta D$ & Change in power over change in duty ratio \\
\hline STC & Standard test conditions \\
\hline INC & Incremental Conductance \\
\hline MINC & Modified Incremental Conductance \\
\hline CNM & Classical Numerical Methods \\
\hline MCNM & Modified Classical Numerical Methods \\
\hline BSM & Bisection Search Method \\
\hline RFM & Regula Falsi Method \\
\hline NRM & Newton Raphson Method \\
\hline SM & Secant Method \\
\hline BNM & Brent Numerical Method \\
\hline MBSM & Modified Bisection Search Method \\
\hline MRFM & Modified Regula Falsi Method \\
\hline MNRM & Modified Newton Raphson Method \\
\hline MSM & Modified Secant Method \\
\hline MBNM & Modified Brent Numerical Method \\
\hline HT & Hybrid Techniques \\
\hline
\end{tabular}

\section{Introduction}

Solar energy attains the most credible position amongst all the available renewable energy sources due to its reliability and cleanliness [1-3]. However, installation expenses, dependency on weather condition and lowefficiency, remain the steep drawbacks of solar energy. In order to confront such issues, maximum power point tracking techniques have been proposed by researchers to extract maximum output from the PV arrays. Considering the PV curve, the point offering optimum power remains MPP [4]. Different MPPT techniques with a categorical digital and analog classification has been presented by researchers in [5]. 
Steady-state oscillations and slow transient response, remain two of the most common issues for many of the conventional MPPT techniques [6]. Moreover, CNM have also been utilized for DMPPT of PV systems [5, 7-10]. Shortcomings of such techniques, as algorithm numerical stability, discretization error and quantization error have been explored in [10]. However, the issues confronted while implementing DMPPT for various Dc-Dc converter topologies, limited range of operation, PV array dependence and accuracy of the initial guess have not been addressed. This paper addresses such concerns by proposing HT and theoretically analysing the conditions for optimum performance of DMPPT by HT on various converter topologies. The HT offers lower computational complexity, faster dynamic response, easy implementation, PV array independence, accuracy of initial guess and fewer overshoots.

Considering the problems of CNM, an amalgamation of MCNM and MINC has been utilized to realize the HT. As, the bisection search method (BSM) is reliable, yet converges slowly. Reliability of BSM is offset by its disappointing linear convergence. Moreover, it typically involves $\log _{2} \frac{\mathrm{b}-\mathrm{a}}{\delta}$ iterations in order to attain a certain accuracy tolerance $\delta[11,12]$. Further, Newton-Raphson method (NRM) remains much more efficient than BSM. However, calculation of derivative is required by NRM, which adds to its complexity [13]. In certain cases, if initial guess is too far away from the root, the NRM may not converge. This causes tangent line offshoot. However, it remains faster than BSM. By contrast, Secant Method (SM) is quick at convergence, but may diverge without reliable initial guesses [5]. Furthermore, Brent's method usually converges quickly to a root, yet for occasional difficult functions, it generically requires $\mathrm{O}(\mathrm{n})$ or $\mathrm{O}\left(\mathrm{n}^{2}\right)$ number of iterations to find a root; $\mathrm{n}$ being the number of steps required by BSM for convergence [11]. Moreover, as observed in [10] all the numerical methods for MPPT application require predefined information for the initial guesses and closed bracketed limited. Therefore, we present HT that places a stricter bound on the search for the MPP. Here, DMPPT $[6,14,15]$ is utilized instead of MPPT because all the CNMs and MCNMs under discussion have been implemented digitally (on DSP) and previous work on MPPT by CNMs is also reported to be digitally implemented $[6,10,14]$.

The objectives of this paper are as follows:

1. Highlight the issues confronted while implementing DMPPT for various Dc-Dc converter topologies and theoretically analyse the conditions for optimum performance.

2. Present HT with direct control offering a combination of the MINC and MCNM techniques. In addition, practically and theoretically prove the improved performance of the proposed techniques.

3. Provide comparative analysis of the HT and the MINC direct control technique. 
The paper is structured as follows: Section 2 introduces the DMPPT and the shortcomings in implementing DMPPT on various Dc-Dc converters. Subsequently, section 3 presents an overview of all the MCNM under consideration and investigates the combination of the MINC MPPT Technique with the MCNM to offer the proposed Hybrid DMPPT techniques. Further, section 4 presents simulation and experimental results for all the MPPT techniques under consideration. To further validate the outcomes, section 5 offers a discussion highlighting the improved performance of HT in terms of lower computational complexity, faster dynamic response, easy implementation, PV array independence, accuracy of initial guess and fewer overshoots. Lastly, conclusion of this work is offered in Section 6.

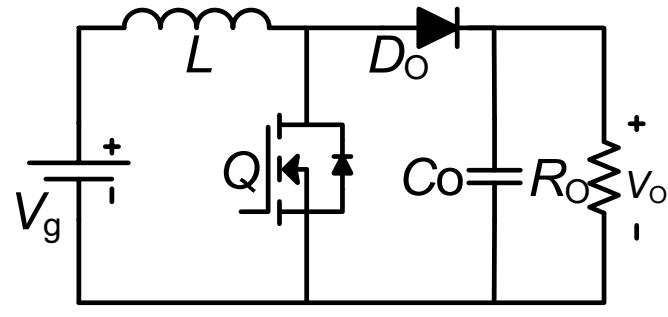

(a) Conventional Boost Converter

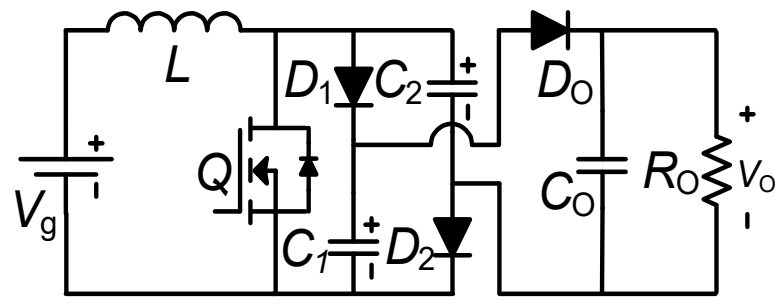

(c) 2-SSC Boost Converter

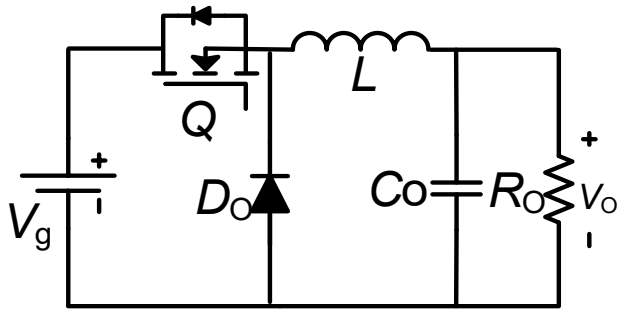

(b) Conventional Buck Converter

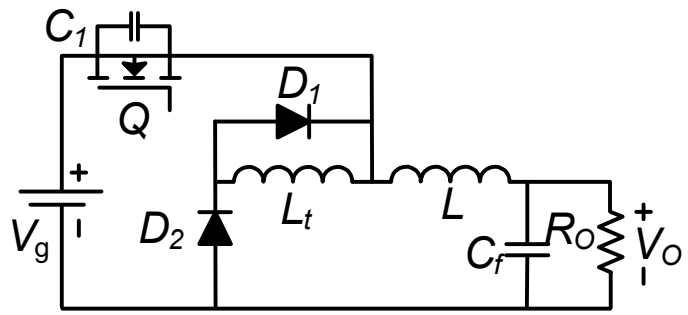

(d) Optimized Buck Converter.

Figure. 1. Dc-Dc converter topologies.

\section{Shortcomings of DMPPT implementation on various Dc-Dc converter topologies}

Considering direct control MPPT techniques, duty cycle (D) is taken as the main control variable. Therefore, performances of DMPPT techniques with direct control, show a trade-off between the transient response and the steady state error. Here, the primary issue remains that a constant voltage change is never guaranteed with a constant step size change in duty cycle.

A PV system can employ various Dc-Dc converter topologies. Here, the focus remains on the OBC [16] and 2SSC Boost converter. In case of a PV system employing the OBC, at operating points away from the MPP the 
system shows smaller steady state oscillations [17], as a content step change offers small change in voltage, however, at operating points closer to MPP large steady state response is observed, as the constant step change offers large change in voltage. For Boost converter or 2-SSC Boost converter the conditions are entirely opposite to the ones observed for OBC. Fig. 1 presents the various Dc-Dc converter topologies utilized and Table 1 presents the system parameters for OBC and 2-SSC Boost Converter.

\begin{tabular}{|c|c|c|}
\hline Table. 2 System parameters & Optimized Buck Converter \\
\hline Parameters & 2-SSC Boost Converter & $20 \mathrm{kHz}$ \\
\hline Switching frequency & $20 \mathrm{kHz}$ & $0.1 \mathrm{~s}$ \\
\hline Sampling time & $0.1 \mathrm{~s}$ & $2200 \mu \mathrm{F}$ \\
\hline Input capacitor & $2200 \mu \mathrm{F}$ & - \\
\hline Switch capacitor & $1 \mu \mathrm{F}$ & $560 \mu \mathrm{H}$ \\
\hline Inductor (L) & $560 \mu \mathrm{H}$ & $0.5 \mu \mathrm{H}$ \\
\hline Inductor $(L t)$ & - & $220 \mu \mathrm{F}$ \\
\hline Filter capacitor & $220 \mu \mathrm{F}$ & $1 \Omega$ \\
\hline Load & $15 \Omega$ & \\
\hline
\end{tabular}

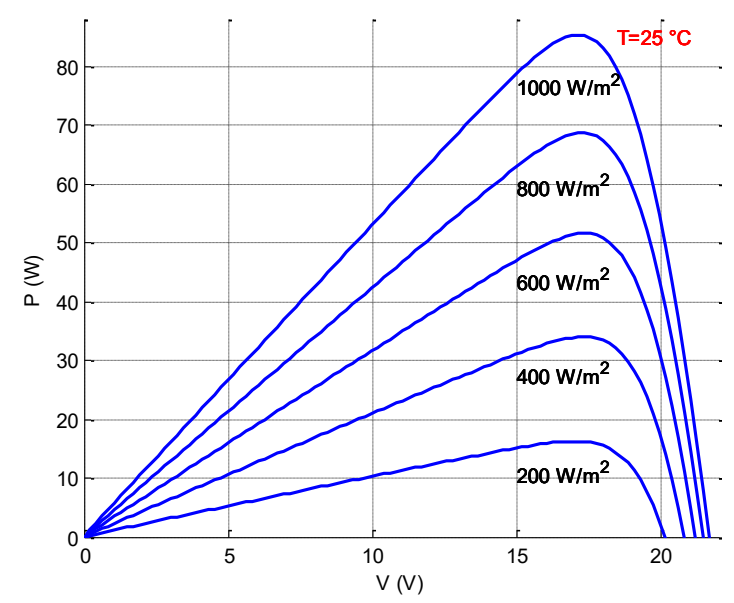

Figure. 2. P-V curves under irradiance change.

Kyocera KC85T PV panel has been employed to validate the effectiveness of the proposed DMPPT algorithms. Table 2 offers the PV performance parameters. Fig. 2 presents the power versus voltage $(P-V)$ plots for the PV module under different irradiance conditions.

Table. 2 performance parameters for the PV Module

\begin{tabular}{|c|c|}
\hline Table. 2 PV Module \\
\hline Parameters & Value \\
\hline Vmpp & $17.4 \mathrm{~V}$ \\
\hline Voc & $21.7 \mathrm{~V}$ \\
\hline Impp & $5.02 \mathrm{~A}$ \\
\hline
\end{tabular}




\begin{tabular}{|c|c|}
\hline Isc & $5.34 \mathrm{~A}$ \\
\hline Pmpp & $87 \mathrm{~W}$ \\
\hline Series cells & 36 \\
\hline
\end{tabular}

\subsection{Change in Voltage for High Duty Cycle}

\subsubsection{Boost Converter}

Fig. 1(a) presents the schematic diagram of the conventional boost converter. The effective resistance seen by the source for the boost converter can be presented as:

$$
R_{p v}=\frac{V_{p v}}{I_{p v}}=R(1-D)^{2}
$$

As this region of operation can be considered as the constant current region so:

$$
V_{p v}=R(1-D)^{2} I_{s c}=k(1-D)^{2}
$$

where, $R I_{s c}=k$

Differentiating (2) with respect to $D$, convergence rate of $V_{p v}$ can be attained as

$$
\frac{\Delta V_{p v}}{\Delta D}=(2 D-2) k=2(D-1) k
$$

As observed from equation (3), the change in voltage for Boost converter establishes a direct proportionality with duty cycle. Hence, the direction of convergence for both the input voltage and duty cycle remains same. As D is slightly reduced a considerable change in voltage is observed. But, as D continues to decrease the change lessens.

\subsubsection{2-Stage High Boost converter}

Similarly, for 2-SSC the effective resistance can be realized as,

$$
R_{p v}=\frac{V_{p v}}{I_{p v}}=\frac{R(1-D)^{2}}{4}
$$

Convergence rate of $V_{p v}$ can be attained as

$$
\frac{\Delta V_{p v}}{\Delta D}=\frac{(D-1)}{2} k
$$

Similarly, as observed from equation (5), the change in voltage for 2-SSC Boost converter establishes a direct proportionality with duty cycle. However, the change in voltage will be less than that observed with conventional boost convereter. 


\subsubsection{Optimum Buck converter}

Fig. 1. (d) presents the design configuration of the OBC. The mathematical modelling of OBC can be done as presented in [16]. Here, DMPPT with direct implementation has been utilized. In order to compensate the hard switching losses such a converter has been employed. As, it offers minimum voltage and current stresses, less components and PWM control with soft switching utilizing only one switch.

For OBC the effective resistance can be realized as,

$$
R_{p v}=\frac{V_{p v}}{I_{p v}}=\frac{R}{D^{2}}
$$

Convergence rate of $V_{p v}$ can be attained as

$$
\frac{\Delta V_{p v}}{\Delta D}=-\frac{2 K}{D^{3}}
$$

As observed from equation (7), the change in voltage for $\mathrm{OBC}$ establishes an inverse proportionality with duty cycle. Hence, the direction of convergence for the input voltage and duty cycle remains opposite. As D is slightly reduced a small change in voltage is observed. But, as D continues to decrease the change increases.

\subsection{Change in Voltage for Duty Cycle Closer to MPP}

\subsubsection{Boost Converter}

As it is evident that

$$
\frac{\Delta P_{p v}}{\Delta D}=\frac{\Delta I_{p v} V_{p v}}{\Delta D}-\frac{I_{p v} \Delta V_{p v}}{\Delta D}
$$

At MPP:

$$
\frac{\Delta P_{p v}}{\Delta D}=0
$$

Therefore,

$$
0=\frac{\Delta I_{p v} V_{p v}}{\Delta D}-\frac{I_{p v} \Delta V_{p v}}{\Delta D}
$$

Change in voltage for duty cycle closer to MPP can be ascertained by,

$$
I_{p v}=\frac{1}{R(1-D)^{2}} V_{p v}
$$

Differentiating (11) with respect to D 


$$
\frac{\Delta I_{p v}}{\Delta D}=\frac{1}{R}\left[\frac{(1-D)^{2} \frac{\Delta V p v}{\Delta D}-(-2+2 D) V_{p v}}{(1-D)^{4}}\right]
$$

Substituting equation (12) into (10) we get

$$
V_{p v}\left(\frac{1}{R}\left[\frac{(1-D)^{2} \frac{\Delta V_{p v}}{\Delta D}-(-2+2 D) V_{p v}}{(1-D)^{4}}\right]\right)+\frac{I_{p v} \Delta V_{p v}}{\Delta D}=0
$$

Further,

$$
\frac{\Delta V_{p v}}{\Delta D}=\frac{(-2+2 D) V_{p v}^{2}}{\left(\frac{V p v}{(1-D)^{2}}+R I_{p v}\right)(1-D)^{4}}
$$

At MPP,

$$
\frac{\Delta V_{p v}}{\Delta D}=\frac{(-2+2 D) V_{M P P}^{2}}{\left(\frac{V_{M P P}}{(1-D)^{2}}+R I_{M P P}\right)(1-D)^{4}}
$$

Substituting equation (1) into (15) we get,

$$
\begin{aligned}
& \frac{\Delta V_{p v}}{\Delta D}=\frac{(-2+2 D) V_{M P P}^{2}}{2 V_{M P P}(1-D)^{2}} \\
& \frac{\Delta V_{p v}}{\Delta D}=\frac{(D-1) V_{M P P}}{(1-D)^{2}}
\end{aligned}
$$

\subsubsection{2-Stage High Boost Converter}

Similarly, for 2-SSC the change in voltage with respected to D, closer to MPP can be realized as,

$$
\frac{\Delta V_{p v}}{\Delta D}=\frac{(D-1) V_{M P P}}{(1-D)^{2}}
$$

\subsubsection{Optimum Buck Converter}

For OBC the change in voltage with respected to D, closer to MPP can be realized as,

$$
\frac{\Delta V_{p v}}{\Delta D}=\frac{-V_{M P P}}{D}
$$

Considering equations $(17,18$ and 19) it is clear that at MPPT where the change in change in power with respect to change in duty cycle is almost equal to 0 , the response of input voltage remains fast for $\mathrm{OBC}$ and faster for Boost and 2-SSC Boost Converter. As at MPP, D is certainly less than 1 and voltage at MPP is optimum. Hence, the change of voltage for all converters remains large. Notable is the fact that at MPP conventional Boost and 2SSC Boost converter operate in a similar fashion. 


\subsubsection{Boost Converter}

Change in voltage for duty cycle away from MPP with low D can be ascertained by differentiating equation (1),

$$
\frac{\Delta V_{p v}}{\Delta D}=R \frac{\Delta}{\Delta D}\left(I_{p v}(1-D)^{2}\right)=R\left(\frac{\Delta I_{p v}}{\Delta D}(1-D)^{2}+(-2+2 D) I_{p v}\right)
$$

Substituting $I_{p v}$ into equation (20) we get,

$$
\frac{\Delta V_{p v}}{\Delta D}=R\left(\frac{\Delta I_{p v}}{\Delta D}(1-D)^{2}\right)+\frac{(2 D-2) V_{p v}}{(1-D)^{2}}
$$

From equations (3) and (21), the directions of input voltage and the Duty cycle remain the same, so the change in voltage is essentially positive. Therefore, at low Duty cycle the change in voltage is high.

\subsubsection{2-Stage High Boost Converter}

Similarly, for 2-SSC the change in voltage with respected to D, away from MPP at low D can be realized as,

$$
\frac{\Delta V_{p v}}{\Delta D}=\frac{R}{4}\left(\frac{\Delta I_{p v}}{\Delta D}(1-D)^{2}\right)+\frac{4(2 D-2) V_{p v}}{(1-D)^{2}}
$$

From equations (5) and (22), the directions of input voltage and the Duty cycle remain the same, so the change in voltage is essentially positive and higher than the conventional boost converter.

\subsubsection{Optimum Buck Converter}

For OBC the change in voltage with respected to D, at low $\mathrm{D}$ can be realized as,

$$
\frac{\Delta V_{p v}}{\Delta D}=\frac{\Delta I_{p v}}{\Delta D}\left(\frac{R}{D^{2}}\right)-\frac{2 V_{p v}}{D}
$$

From equations (7) and (23), the directions of input voltage and the Duty cycle remain opposite, so the change in

voltage is essentially negative. As the term $\frac{\Delta I_{p v}}{\Delta D}\left(\frac{R}{D^{2}}\right)$ is smaller than $\frac{2 V_{p v}}{D}$. Therefore, at low Duty cycle the change in voltage is small and negative.

\section{Modified Numerical Methods}

\subsection{Close Bracket Methods}

\subsubsection{Modified Bisection Search Method (MBSM)}


The simplest root-finding algorithm is BSM $[12,13]$. It requires the knowledge of two initial guesses. Moreover, BSM remains applicable on continues functions. Considering, a and $b$, as initial guesses, such that $f(a)$ and $f(b)$ have opposite signs. For BSM a midpoint between $[\mathrm{a}, \mathrm{b}]$ is calculated, and determined whether root lies on the [a, $(a+b) / 2]$ or $[(a+b) / 2, b]$ point. This loop is iterated until the interval is sufficiently small.

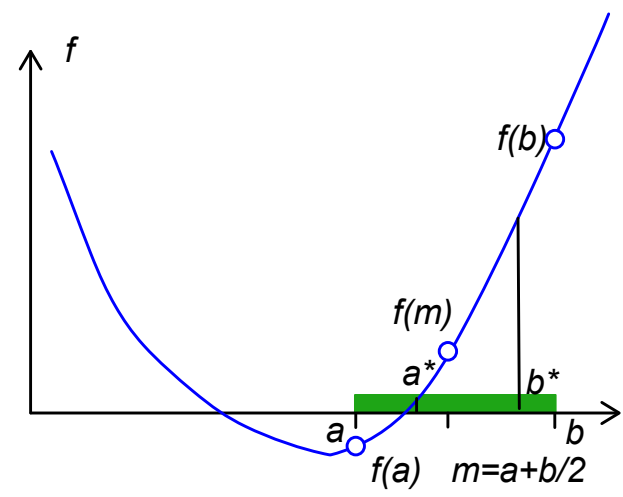

Figure. 3. MBS Method

For MBSM a subinterval is introduced. The algorithm represented in Fig. 3 can be summarized as [18]:

1) Set an integer $k=1$, a tolerance limit $\delta$ and $a_{k}=a, b_{k}=b$; where $f(a) f(b)<0$.

2) Compute $m=\left(\frac{a_{k}+b_{k}}{2}\right)$

3) Compute for a subinterval $\left(a_{k}^{*}, b_{k}^{*}\right)$ by

$\left(a_{k}^{*}, b_{k}^{*}\right)=\left\{\begin{array}{l}\left(a_{k}, m_{k}\right), \text { if } f\left(a_{k}\right) f\left(m_{k}\right)<0 \\ \left(m_{k}, b_{k}\right), \text { if } f\left(m_{k}\right) f\left(b_{k}\right)<0\end{array}\right\}$

Compute for $x_{k}=-\frac{m}{c}$, where $c=\frac{f\left(\mathrm{~b}_{\mathrm{k}}^{*}\right)-f\left(\mathrm{a}_{\mathrm{k}}^{*}\right)}{\mathrm{b}_{\mathrm{k}}^{*}-\mathrm{a}_{\mathrm{k}}^{*}}, m=f\left(\mathrm{~b}_{\mathrm{k}}^{*}\right)-c \cdot \mathrm{b}_{\mathrm{k}}^{*}$ or $m=f\left(\mathrm{a}_{\mathrm{k}}^{*}\right)-c \cdot a_{\mathrm{k}}^{*}$

4) If $\left|f\left(x_{k}\right)\right|<\delta$, End

Else $\left(a_{k+1}, b_{k+1}\right)=\left\{\begin{array}{l}\left(a_{k}^{*}, x_{k}\right), \text { if } f\left(a_{k}^{*}\right) f\left(x_{k}\right)<0 \\ \left(x_{k}, b_{k}^{*}\right), \text { if } f\left(x_{k}\right) f\left(b_{k}^{*}\right)<0\end{array}\right\}$

And set $\mathrm{k}=\mathrm{k}+1$, Goto step 2 .

\subsubsection{Modified Regula Falsi Method (MRFM)}

The RFM is an amalgamation of BSM and Secant Method (SM) [10]. It remains linearly convergent. 


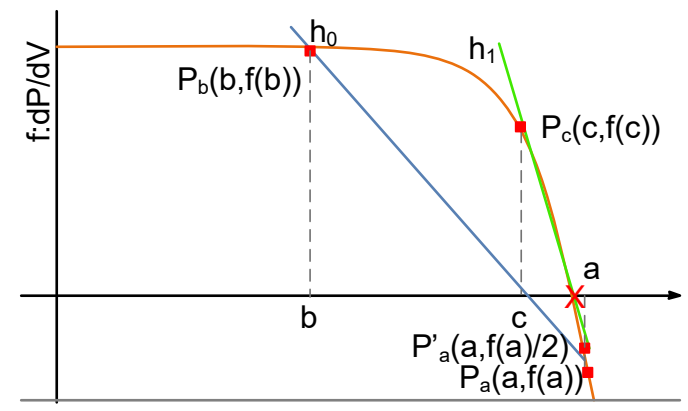

Figure. 4. MRF Method

MRFM is almost similar to RFM. However, the algorithm differs in the following steps as represented in Fig. 4 and in [9], where $h_{0}$ and $h_{1}$ are the tangents a and $b$ are the initial guesses and $f(a)$ and $f(b)$ the corresponding functions:

1) Set an integer $k=1$, a tolerance limit $\delta$ and $a_{k}=a, b_{k}=b$; where $f(a) f(b)<0$.

IF $\mathrm{f}\left(b_{k}\right) \mathrm{f}\left(a_{k}\right)<0$ and $\mathrm{f}\left(b_{k}\right)>0, T H E N$

$c_{k}=\left(\frac{\mathrm{b}_{\mathrm{k}} \cdot \mathrm{f}\left(a_{k}\right) \cdot 0.5-\mathrm{a}_{\mathrm{k}} \cdot \mathrm{f}\left(\mathrm{b}_{k}\right)}{0.5 \cdot \mathrm{f}\left(a_{k}\right)-\mathrm{f}\left(\mathrm{b}_{\mathrm{k}}\right)}\right)$

IF $\mathrm{f}\left(b_{k}\right) \mathrm{f}\left(a_{k}\right)<0$ and $\mathrm{f}\left(b_{k}\right)<0, T H E N$

$c_{k}=\left(\frac{\mathrm{b}_{\mathrm{k}} \cdot \mathrm{f}\left(a_{k}\right)-\mathrm{a}_{\mathrm{k}} \cdot \mathrm{f}\left(\mathrm{b}_{k}\right) \cdot 0.5}{\mathrm{f}\left(a_{k}\right)-0.5 \cdot \mathrm{f}\left(\mathrm{b}_{\mathrm{k}}\right)}\right)$

2) If $\left|f\left(c_{k}\right)\right|<\delta$, End

$I F \mathrm{f}\left(c_{k}\right) \mathrm{f}\left(a_{k}\right)<0$ THEN $b_{k}=c_{k}$

$\operatorname{ELSEIF} \mathrm{f}\left(c_{k}\right) \mathrm{f}\left(b_{k}\right)<0$ THEN $a_{k}=c_{k}$

And set $\mathrm{k}=\mathrm{k}+1$ for the next iteration.

\subsection{Open Bracket Methods}

\subsubsection{Modified Newton-Rapshon Method (MNRM)}

The NRM is a linearly convergent method and its iterative approach has been detailed in $[5,7,12,13]$. In order to attain a quadratic convergence, MNRM provides modifications to NRM as presented in Fig. 5 and [13]:

$x_{n+1}=x_{n}-\frac{f\left(x_{n}\right) f^{\prime}\left(x_{n}\right)}{\left[f^{\prime}\left(x_{n}\right)\right]^{2}-f\left(x_{n}\right) f^{\prime \prime}\left(x_{n}\right)}$

$\operatorname{Until}\left|f\left(c_{k}\right)\right|<\delta$. 


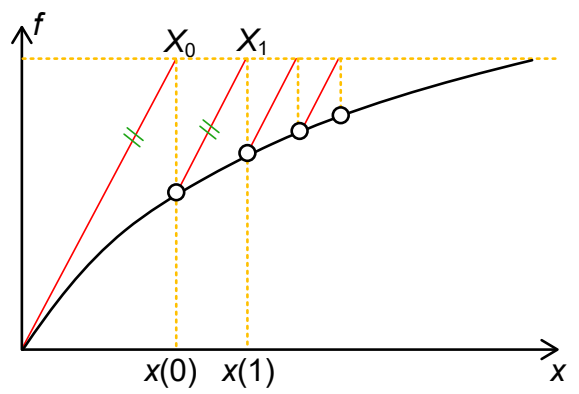

Figure. 5. MNR Method

In practical implementations, NRM employed for MPPT would probably diverge or be numerically unstable [5].

\subsubsection{Modified Secant Method (MSM)}

SM approach requires two initial estimates. SM is in many ways similar to NRM. It offer an ease in order to reduce the necessity of utilizing two arbitrary values to estimate the derivative. To calculate $f^{\prime}\left(x_{n}\right)$ as presented in Fig. 6 and [13] MSM involves a fractional perturbation of the independent variable.

$x_{n+1}=x_{n}-\frac{\xi x_{n} f\left(x_{n}\right)}{f\left(x_{n}+\xi x_{n}\right)-f\left(x_{n}\right)}$

Until $\left|f\left(c_{k}\right)\right|<\delta . \xi$ a small perturbation fraction.

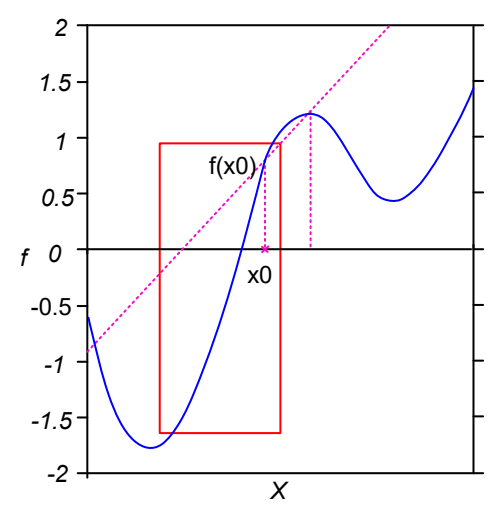

Figure. 6. MS Method

MSM provides a nice means to attain the efficiency of the NRM without having to compute derivatives. 


\subsection{Combination of Numerical Methods}

\subsubsection{Modified Brent Numerical Method (MBNM)}

BM utilizes the more precise solution between SM or BSM or the inverse quadratic interpolation [19]. It offers the speed of SM and reliability of BSM. For MBNM, a bisection step should be forced under the following circumstances as presented in Fig. 7 and [11]:

1) If five successive interpolation steps fail to reduce the size of the original interval or the last interval generated by a bisection step by a factor of two.

2) If an interpolation step produces a point, $b$, such that $|f(b)|$ is not at least a factor of two smaller than the previous best point.

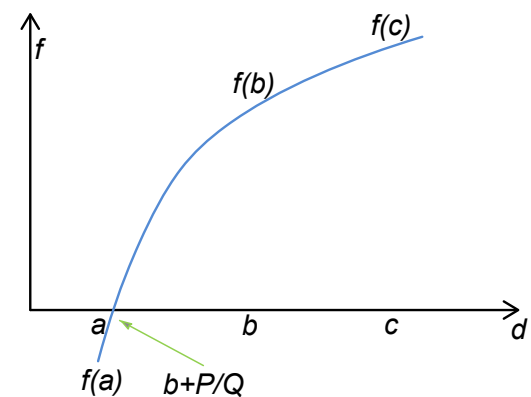

Figure. 7. MBN Method. 


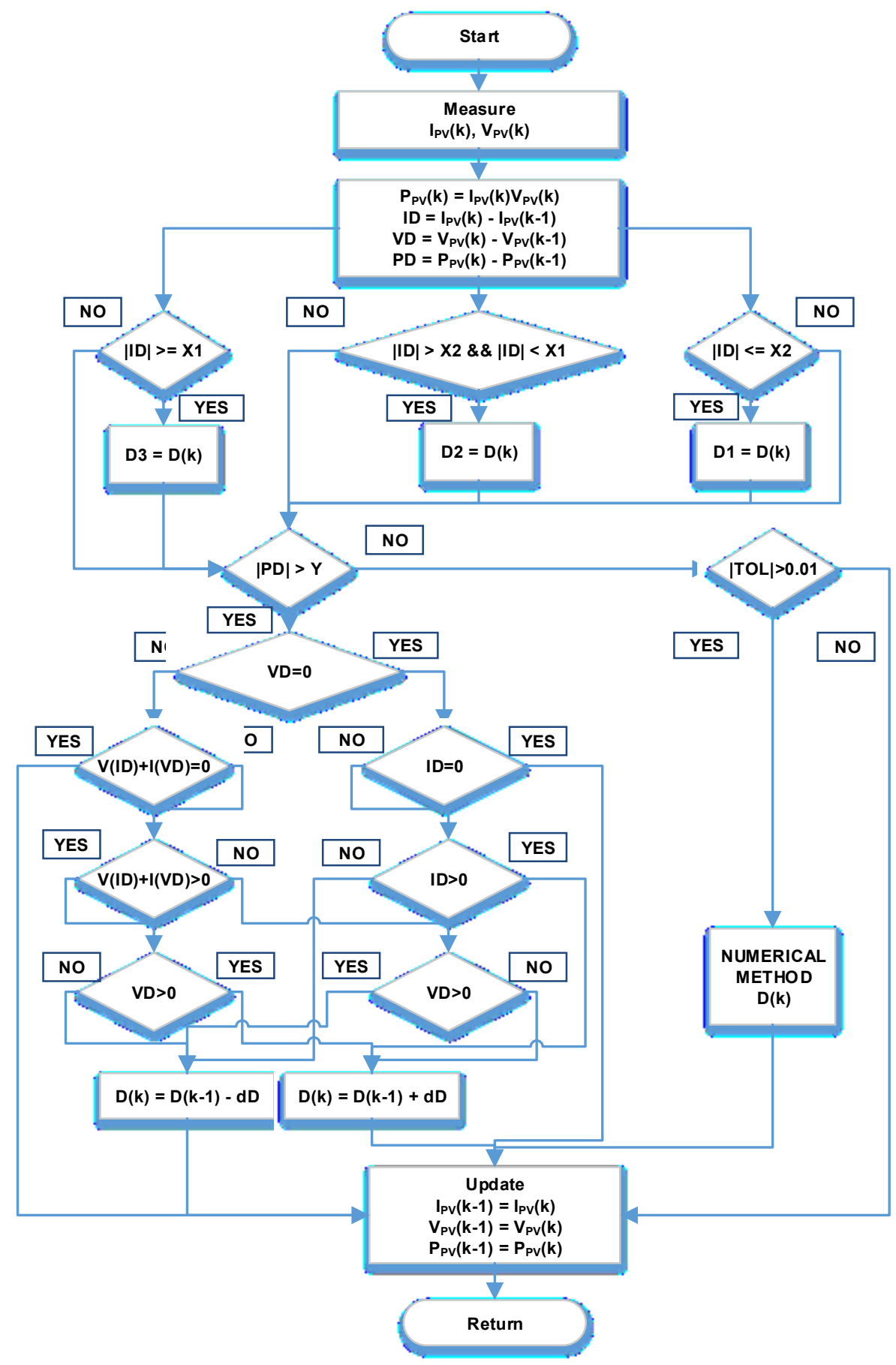

Figure. 8. Flowchart of the HT with 2-Stage Switch Capacitor based Boost Converter 


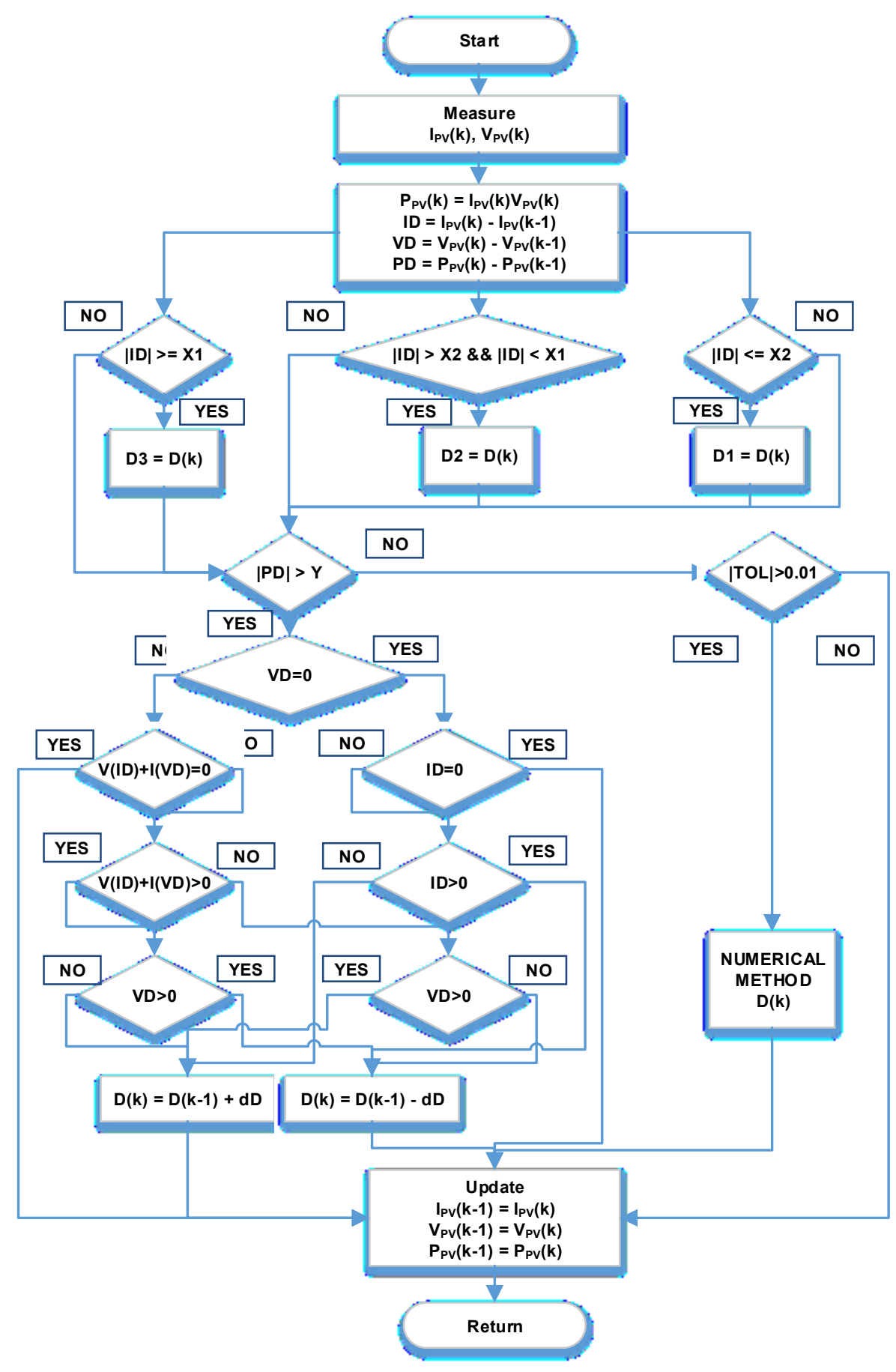

Figure. 9. Flowchart of the HT with Optimum Buck Converter

Fig. 8 presents the flowchart for the direct control MINC method [17] and MCNM for Boost converter, whereas, Fig. 9 presents the flowchart for operation of HT on OBC. The initial guess need not be determined by mathematical modeling, but it is taken by the HT based on the PV current. As, change in current $(\Delta \mathrm{I})$ is trivial, in 
the constant current region. Therefore, MPP operational point can be estimated in such a region. Moreover, when considering a predefined tolerance error $(e)$, such a point is obtained when $\Delta \mathrm{I}$ is marginally lower than $e$, as:

$\Delta I<e$

Here, the step size is the least. Moreover, because of least perturbation step at MPP, choice for $e$ is to be lesser than current changes and greater than zero, as:

$0<e<\Delta I$

Therefore the change in current is employed for determining the initial guesses. The limits of $\Delta I$ for HT have been placed by trial and error method. The HT operates the numerical method once it is in the $\Delta I$ limits mentioned, else it utilizes the MINC.

\section{RESULTS}

\subsection{SIMULATION RESULTS:}

Matlab/Simulink software was utilized to simulate the performances of the MINC and HT. Table 1 and 2 present the system and PV module parameters utilized for the attaining the simulation results. The MPPT frequency was set to be $0.1 \mathrm{~s}$.

\subsubsection{Simulation results for 2-SSC Boost Converter}
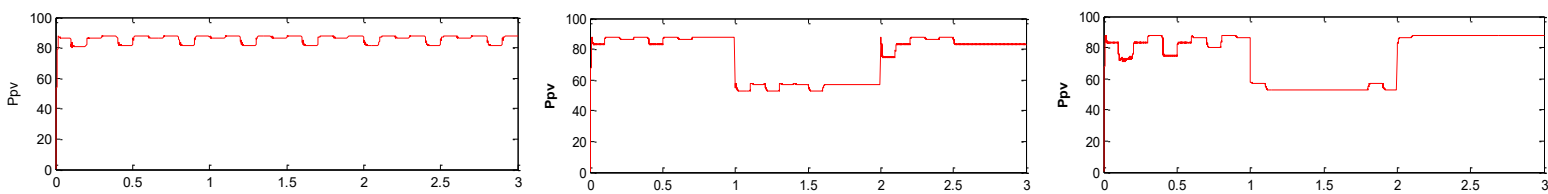

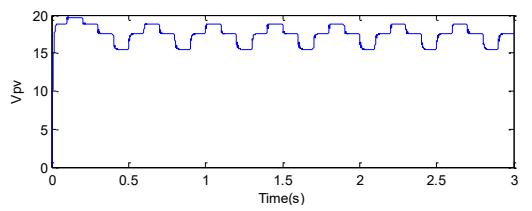

a) $\mathrm{MCINC}$

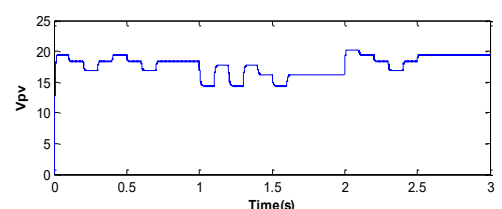

b) HT with MBSM

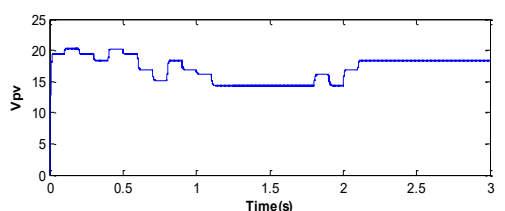

c) HT with MRFM 

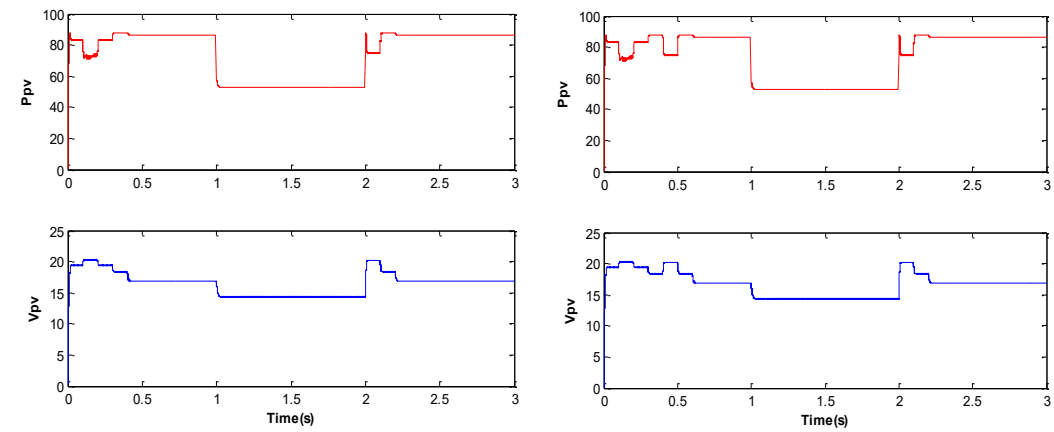

d) HT with MNRM

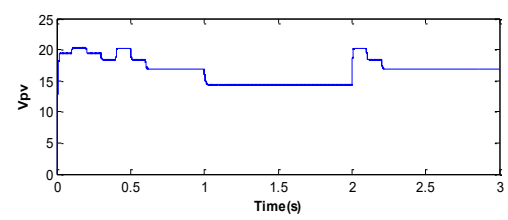

e) HT with MSM
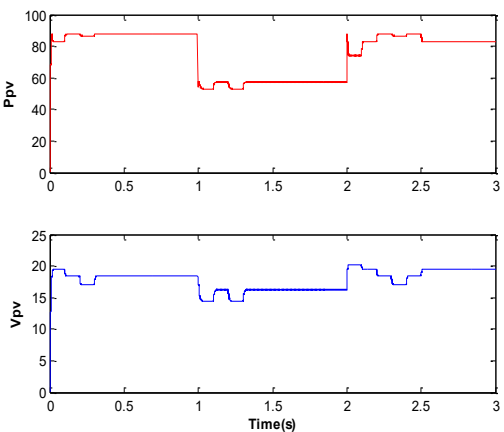

f)

HT with MBNM

Figure. 10. Simulation results for MCINC and HT for 2-SSC Boost Converter

Table. 4

Evaluation of MPPT techniques under discussion implemented on 2-SSC Boost Converter

\begin{tabular}{|c|c|c|c|c|c|}
\hline MPPT Technique & No. of Steps & Settling time & Exact MPP & Actual MPP & Efficiency \\
\hline MINC & - & Oscillating & $87 \mathrm{~W}$ & $84.7 \mathrm{~W}$ & $97.35 \%$ \\
\hline HT with BSM & 8 & $0.8 \mathrm{~s}$ & $87 \mathrm{~W}$ & $86.25 \mathrm{~W}$ & $99.13 \%$ \\
\hline HT with MRF & 9 & $0.9 \mathrm{~s}$ & $87 \mathrm{~W}$ & $86.25 \mathrm{~W}$ & $99.13 \%$ \\
\hline HT with MNR & 5 & $0.5 \mathrm{~s}$ & $87 \mathrm{~W}$ & $86.5 \mathrm{~W}$ & $99.42 \%$ \\
\hline HT with MSM & 6 & $0.6 \mathrm{~s}$ & $87 \mathrm{~W}$ & $86.3 \mathrm{~W}$ & $99.19 \%$ \\
\hline HT with MBNM & 4 & $0.4 \mathrm{~s}$ & $87 \mathrm{~W}$ & $86.5 \mathrm{~W}$ & $99.42 \%$ \\
\hline
\end{tabular}

\subsubsection{Simulation results for $\mathrm{OBC}$}
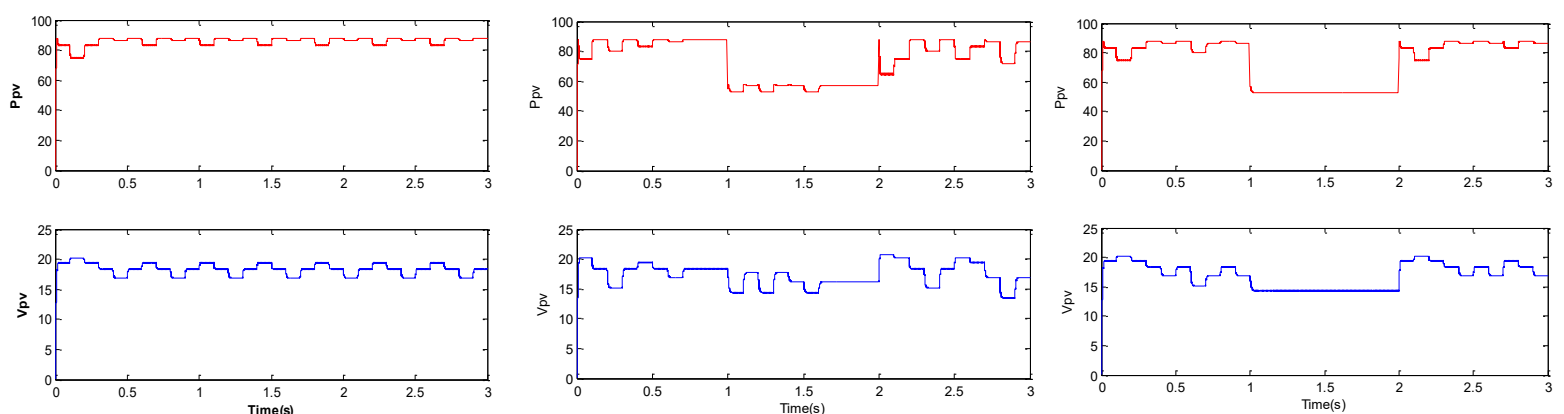

a) $\quad \mathrm{MCINC}$

b) HT with MBSM

c) HT with MRFM
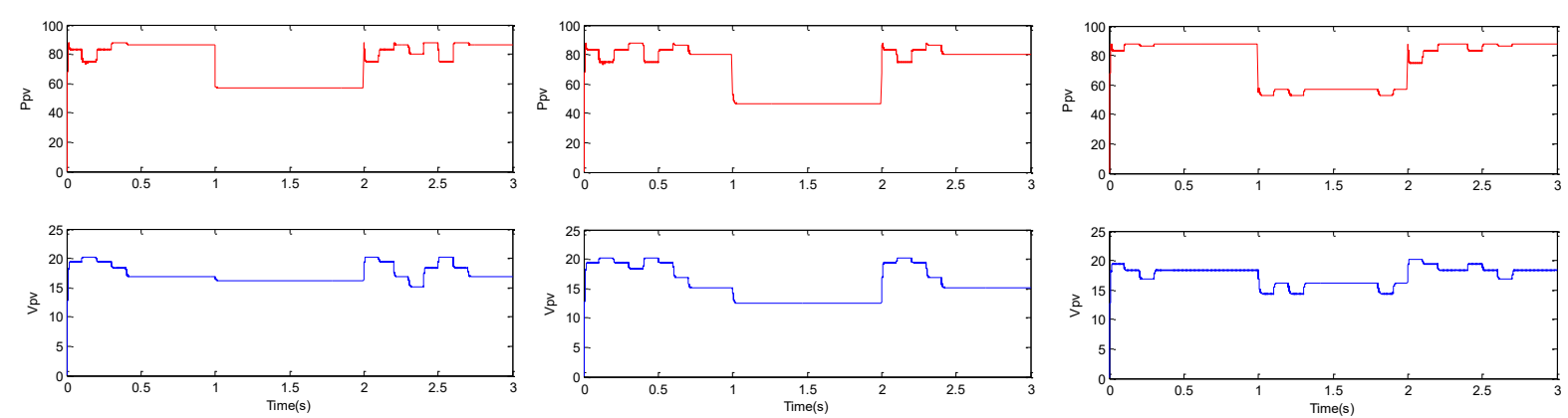

d) $\quad$ HT with MNRM

e) $\quad$ HT with MSM

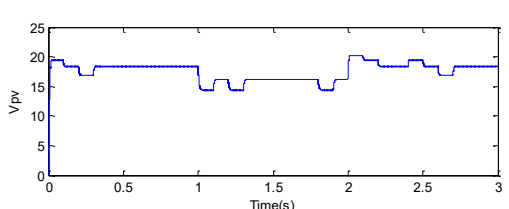

f) HT with MBNM 
Figure. 11. Simulation results for MINC and HT for OBC

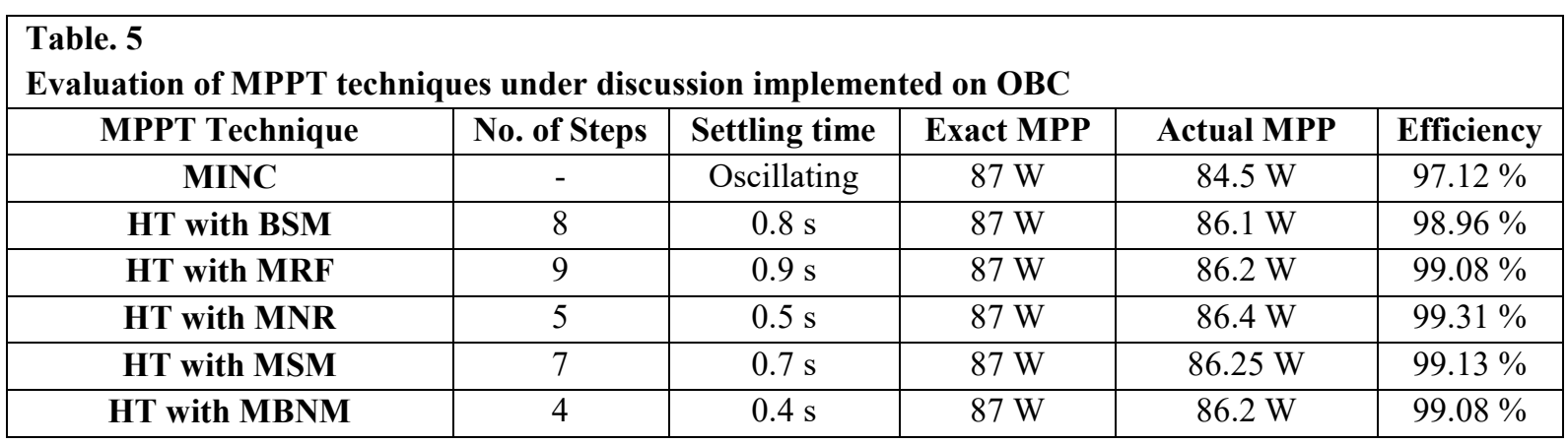

From time $0 \mathrm{~s}$ to $1 \mathrm{~s}$ the results are obtained under STC. Further, the irradiance changes from $1000 \mathrm{~W} / \mathrm{m}^{2}$ to $700 \mathrm{~W} / \mathrm{m}^{2}$ at time $1 \mathrm{~s}$. Finally, the irradiance is set back to $1000 \mathrm{~W} / \mathrm{m}^{2}$ at time $2 \mathrm{~s}$. PV voltage and PV power change for the 2-SSC Boost Converter and OBC of different HT and MINC have been presented in Fig. 10 and 11, respectively. Here, the red plot shows Ppv and the blue plot displays Vpv. As observed, the MINC offers continuous oscillations about the MPP. Further, every mentioned HT remains reliable. Yet, HT with modified open bracket techniques remain faster than the rest. Here, HT with MBNM offers a mediocre speed between the open and close bracketed HT with MCNM. HT with MBSM and MRFM on both converters require almost 8 steps to converge to the MPP. The HT with MNRM entails 5 steps and MSM needs 6 steps. Finally, HT with MBNM requires almost 4 steps for topologies under consideration. The performance of MBNM carries a demerit of less reliability. As, it can offer very fast response at its normal operation, but with sudden changes in irradiance and temperature the operation turns slow due the forced bisection step. Table 4 and 5 present the performance evaluation for simulation results of the MPPT techniques under discussion implemented on 2-SSC Boost Converter and $\mathrm{OBC}$, respectively.

\subsection{Experimental results}

\subsubsection{Ipv and Vpv experimental results}

For experimental results presented in Figs. 12 and 13 the operational time is $50 \mathrm{~s}$ for each method. Here, irradiance is set from $1000 \mathrm{~W} / \mathrm{m}^{2}$ to $700 \mathrm{~W} / \mathrm{m}^{2}$ at time $20 \mathrm{~s}$. Further, at $35 \mathrm{~s}$ the irradiance is set back to $1000 \mathrm{~W} / \mathrm{m}^{2}$. PV voltage has been displayed in yellow, current in blue and power in red of all the mentioned MPPT methods. The plots display the input voltage showing that the open bracketed hybrid techniques for MPPT offer better performance in comparison to closed bracketed. In addition, MBNM offers a performance slightly better than close bracketed techniques. 


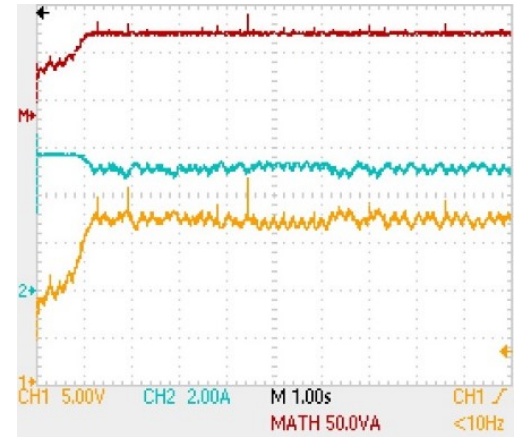

a) $\mathrm{MCINC}$

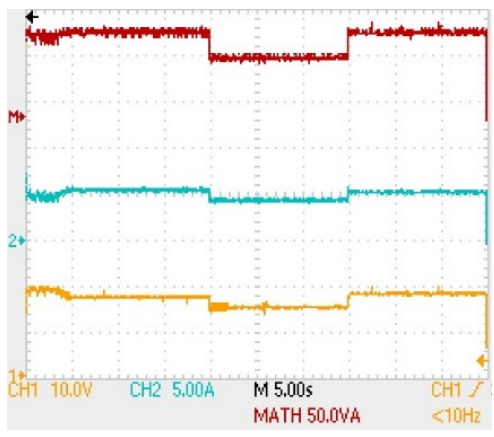

d) HT with NRM

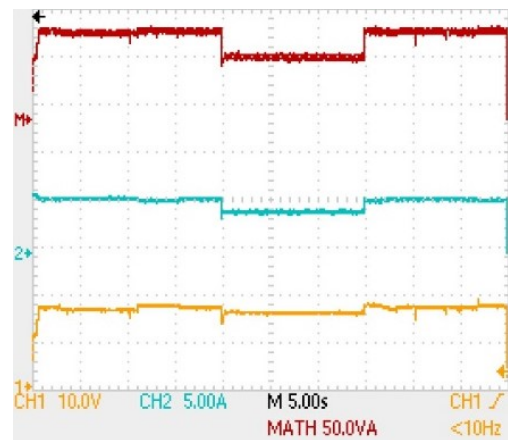

b) HT with MBSM

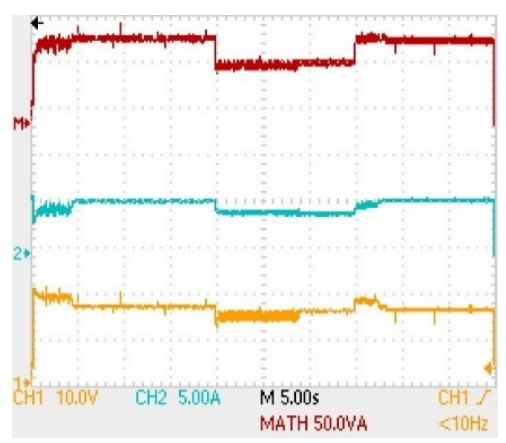

e) HT with MSM

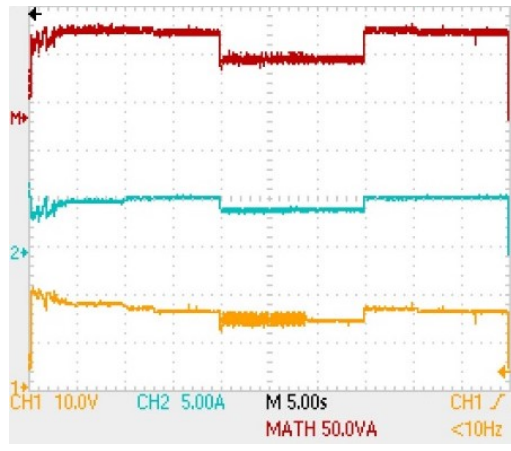

c) HT with MRFM

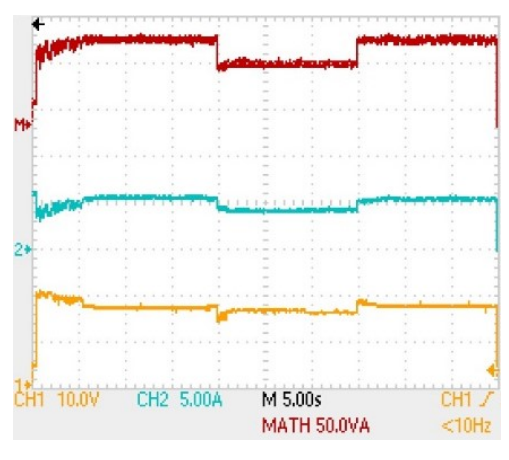

f) HT with MBNM

Figure. 12. Experimental results for MCINC and HT with 2-SSC Boost Converter.

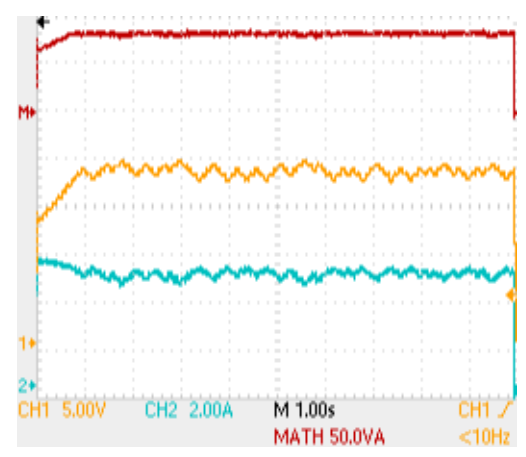

a) $\mathrm{MCINC}$

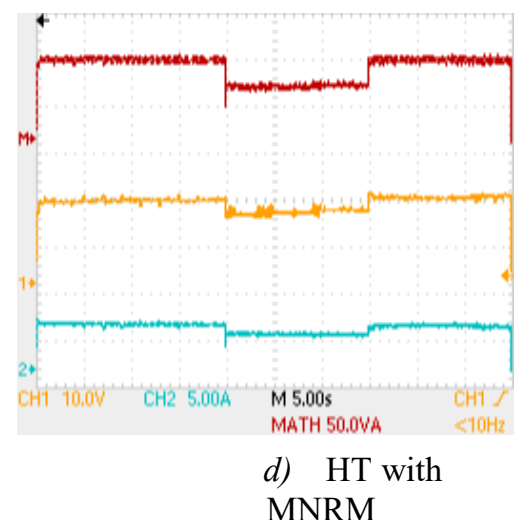

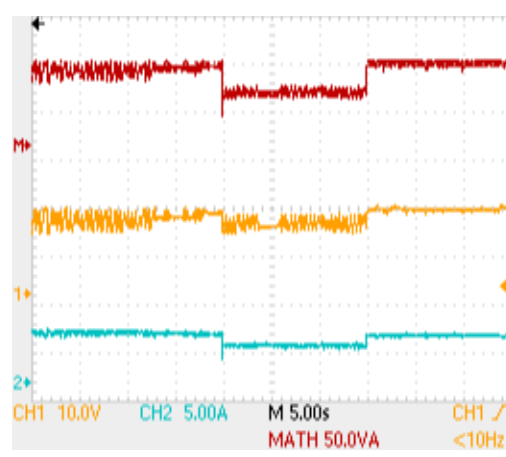

b) HT with MBSM

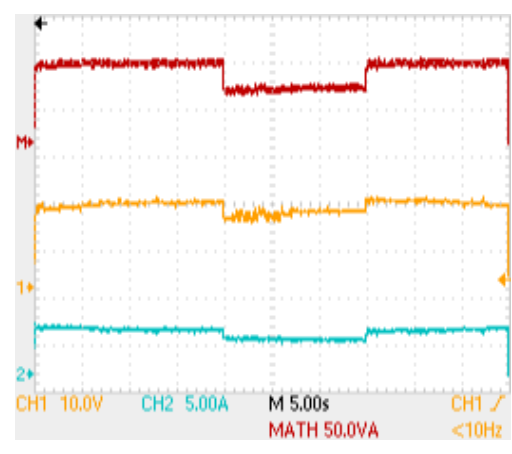

e) HT with MSM

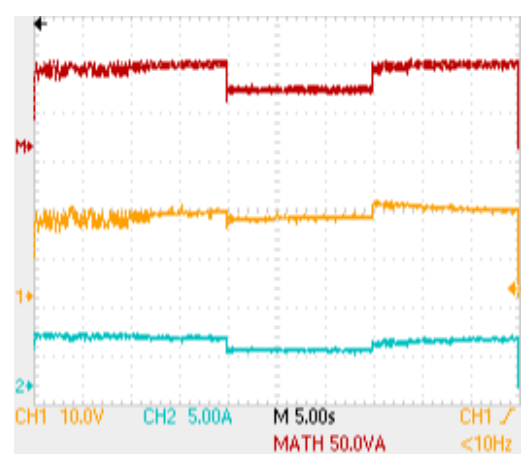

c) HT with MRFM

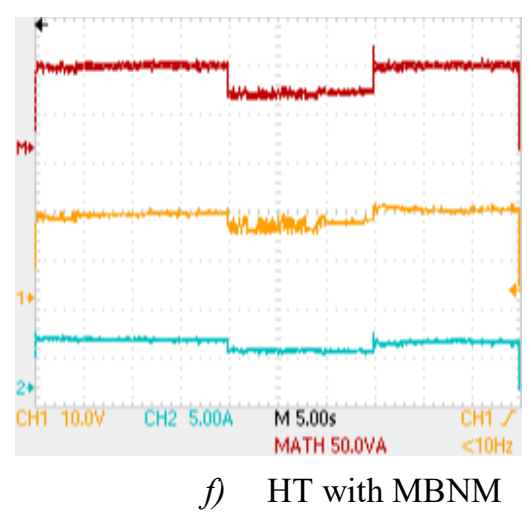

Figure. 13. Experimental results for MCINC and HT with OBC. 


\section{Discussion}

As observed, conventional MPPT techniques including MINC confront issues of slower transient response and steady state oscillation. As presented in Figs. 10, 11, 12 and 13, the performances of HT offer lower computational complexity, faster dynamic response, exact MPP detection, easy implementation, PV array independence, accuracy of initial guess, fewer overshoots, stability and higher MPPT efficiency. Table. 5 presents the overall evaluation of the practical performance of all the techniques under consideration.

\begin{tabular}{|c|c|c|c|}
\hline MPPT technique & Speed & Exact MPP & Complexity \\
\hline MINC & $\mathrm{M}$ & $\mathrm{O}$ & $\mathrm{S}$ \\
\hline HT with BSM & $\mathrm{F}$ & $\mathrm{E}$ & $\mathrm{M}$ \\
\hline HT with MRF & $\mathrm{F}$ & $\mathrm{E}$ & $\mathrm{M}$ \\
\hline HT with MNR & VF & $\mathrm{E}$ & $\mathrm{C}$ \\
\hline HT with MSM & VF & $\mathrm{E}$ & $\mathrm{C}$ \\
\hline HT with MBNM & $\mathrm{F}$ & $\bar{E}$ & $\mathrm{C}$ \\
\hline
\end{tabular}

Note: O - Oscillating, E - Exact MPP, M - Medium, F - Fast, VF - Very Fast, S - Simple, C - Complex.

Analysed parameters are:

As presented in Fig. 9 the HT offers an amalgamation the MINC and MCNM. The hybrid combination offers the following advantages:

\section{A. Limited range of operation}

Direct implementation of CNM confront the issue of limited range of operation. A solution was presented in [10] by modeling the discretization error. However, the range of operation was limited to a few volts. Here the HT determines the range based of the change of current. Moreover, MPP can be tracked even in real weather conditions, with irradiance and temperature changes.

\section{B. PV array dependence}

Utilization of the MCINC in the HT has been the reason PV array independence of the HT. The technique presented in $[9,10]$ required predetermined PV panel knowledge. Whereas, the HT is PV array independent.

\section{Accuracy of the initial guess}

As reported in section 3.4 the HT, for both close and open bracketed methods, considers the constant current region for ascertaining its initial guesses or bracketed limits. As, the initial guess remains in the constant current region and near to the MPP the chances of overshoot, undershoot or incorrect root tracking become very less. 


\section{Exact MPP detection with fewer overshoots}

In comparison to the direct implementation of CNM for MPPT, the HT is easier to implement and offers exact MPP detection. Owing to the close and open bracketed limits initialized in the constant current region, it remains less likely for open bracketed methods to drift away from the root due to a far off initial guesses. Therefore, exact MPP detection with fewer overshoots is assured.

\section{Conclusion}

The HT offers an improved performance for MPPT. As, the HT, due to its combination of MINC and MCNM, attains reliability, accuracy and speed. The shortcomings of CNM have also been addressed, as the HT is PV array independent, determines the range of operation based on real weather conditions and remains accurate in determining the initial guesses so that the numerical methods can operate accurately. Simulation and experimental results present a quite evident picture that the HT offers an improved performance for MPPT. It offer a moderated approach between the conventional MPPT techniques and the numerical methods. It is convenient because of the convergence at the MPP and the faster response as compared to the MINC.

\section{Acknowledgement}

The authors would like to thank the financial and technical assistance provided by the University of Malaya, UM Power Energy Dedicated Advanced Centre (UMPEDAC), Mohe HiCoe - the High Impact Research Grant -(H16001-00-D000032), Fundamental Research Grant Scheme FP065-2014A and Postgraduate Research Fund (PPP) Project Number: PG029-2015B.

\section{References}

[1] H. Fathabadi, "Novel standalone hybrid solar/wind/fuel cell/battery power generation system," Energy, vol. 140, pp. 454-465, 2017/12/01/ 2017.

[2] Y. Luo, L. Zhang, Z. Liu, J. Wu, Y. Zhang, and Z. Wu, "Numerical evaluation on energy saving potential of a solar photovoltaic thermoelectric radiant wall system in cooling dominant climates," Energy, vol. 142, pp. 384-399, 2018/01/01/2018.

[3] M. D. Leonard and E. E. Michaelides, "Grid-independent residential buildings with renewable energy sources," Energy, vol. 148, pp. 448-460, 2018/04/01/2018.

[4] R. Boukenoui, M. Ghanes, J. P. Barbot, R. Bradai, A. Mellit, and H. Salhi, "Experimental assessment of Maximum Power Point Tracking methods for photovoltaic systems," Energy, vol. 132, pp. 324-340, 2017/08/01/ 2017.

[5] A. Amir, A. Amir, J. Selvaraj, and N. A. Rahim, "Study of the MPP tracking algorithms: Focusing the numerical method techniques," Renewable and Sustainable Energy Reviews, vol. 62, pp. 350-371, 2016/09/01/ 2016. 
[6] M. Mao, L. Zhang, P. Duan, Q. Duan, and M. Yang, "Grid-connected modular PV-Converter system with shuffled frog leaping algorithm based DMPPT controller," Energy, vol. 143, pp. 181-190, 2018/01/15/2018.

[7] W. Xiao, M. G. Lind, W. G. Dunford, and A. Capel, "Real-time identification of optimal operating points in photovoltaic power systems," IEEE Transactions on Industrial Electronics, vol. 53, pp. 1017-1026, 2006.

[8] J. Kim and A. Kwasinski, "Maximum power point tracking for multiple photovoltaic modules using rootfinding methods," in Energy Conversion Congress and Exposition (ECCE), 2014 IEEE, 2014, pp. 9-16.

[9] S. Chun and A. Kwasinski, "Modified regula falsi optimization method approach to digital maximum power point tracking for photovoltaic application," in Applied Power Electronics Conference and Exposition (APEC), 2011 Twenty-Sixth Annual IEEE, 2011, pp. 280-286.

[10] S. Chun and A. Kwasinski, "Analysis of classical root-finding methods applied to digital maximum power point tracking for sustainable photovoltaic energy generation," IEEE transactions on power electronics, vol. 26, pp. 3730-3743, 2011.

[11] G. Wilkins and M. Gu, "A modified Brent's method for finding zeros of functions," Numerische Mathematik, vol. 123, pp. 177-188, 2013.

[12] R. L. Burden and J. D. Faires, "Numerical analysis. 2001," Brooks/Cole, USA, 2001.

[13] S. C. Chapra, "Applied Numerical Methods with MATLAB for Engineers and Scientists (2012)," ed: McGraw Hill Publications.

[14] M. Balato, L. Costanzo, and M. Vitelli, "Chapter 5 - DMPPT PV System: Modeling and Control Techniques," in Advances in Renewable Energies and Power Technologies, I. Yahyaoui, Ed., ed: Elsevier, 2018, pp. 163-205.

[15] H. Luo, H. Wen, X. Li, L. Jiang, and Y. Hu, "Synchronous buck converter based low-cost and highefficiency sub-module DMPPT PV system under partial shading conditions," Energy Conversion and Management, vol. 126, pp. 473-487, 2016/10/15/2016.

[16] B. Divakar and D. Sutanto, "Optimum buck converter with a single switch," IEEE transactions on power electronics, vol. 14, pp. 636-642, 1999.

[17] A. Amir, A. Amir, J. Selvaraj, N. A. Rahim, and A. M. Abusorrah, "Conventional and modified MPPT techniques with direct control and dual scaled adaptive step-size," Solar Energy, vol. 157, pp. 1017-1031, 2017/11/15/2017.

[18] S. Tanakan, "A new algorithm of modified bisection method for nonlinear equation," Applied Mathematical Sciences, vol. 7, pp. 6107-6114, 2013.

[19] R. P. Brent, Algorithms for minimization without derivatives: Courier Corporation, 2013. 